\title{
Monitor-While-Drilling - based estimation of rock mass rating with computational intelligence: the case of tunnel excavation front
}

\author{
Marta Galende \\ CARTIF Centro Tecnológico \\ Boecillo, Valladolid, España \\ margal@cartif.es
}

\author{
M.J. Fuente, G.I. Sainz-Palmero \\ Dpto. Ingeniería de Sistemas y Automática \\ Universidad de Valladolid \\ Valladolid, España \\ mjfuente, gresai@eii.uva.es
}

\author{
M. Menéndez \\ VIAS y Construcciones, S.A. \\ Avda. Camino de Santiago 50 \\ 28050 Madrid, España \\ manuel.menedez@vias.es
}

\begin{abstract}
The construction of tunnels has serious geomechanical uncertainties involving matters of both safety and budget. Nowadays, modern machinery gathers very useful information about the drilling process: the so-called Monitor While Drilling (MWD) data. So, one challenge is to provide support for the tunnel construction based on this on-site data .

Here, an MWD based methodology to support tunnel construction is introduced: a Rock Mass Rating (RMR) estimation is provided by an MWD rocky based characterization of the excavation front and expert knowledge [1].

Well-known machine learning (ML) and computational intelligence $(\mathrm{CI})$ techniques are used. In addition, a collectible and "interpretable" base of knowledge is obtained, linking MWD characterized excavation fronts and RMR.

The results from a real tunnel case show a good and serviceable performance: the accuracy of the RMR estimations is high, Error $_{\text {test }} \cong 3 \%$, using a generated knowledge base of 15 fuzzy rules, 3 linguistic variables and 3 linguistic terms.

This proposal is, however, is open to new algorithms to reinforce its performance.
\end{abstract}

Index Terms-Tunneling, RMR, Sofcomputing, Machine Learning, SDBR

\section{INTRODUCTION}

This work is focused on the tunnelling industry, to be precise the specific case of railway tunnels, but this can be applied to other similar cases such as road tunnels; underground mining and utilities, etc. Tunnel excavation has used two main methods: Drill \& Blast and Tunnel Boring Machine (TBM). The first is the most popular excavation method for conventional tunnelling, in particular for railtrack tunnels. In any case, both methodologies involve the use of computer and control based machinery to capture and log data of different natures concerning the process: this is the so-called Monitor or Measurement While Drilling (MWD) data.

This work has been partially supported by the Spanish Ministry of Economy and Competitiveness and European Regional Development Fund (FEDER) through the Project no. DPI2015-67341-C2-2-R and published in Automation in Construction, 93C (2018) pp. 325-338, https://doi.org/10.1016/j.autcon.2018.05.019
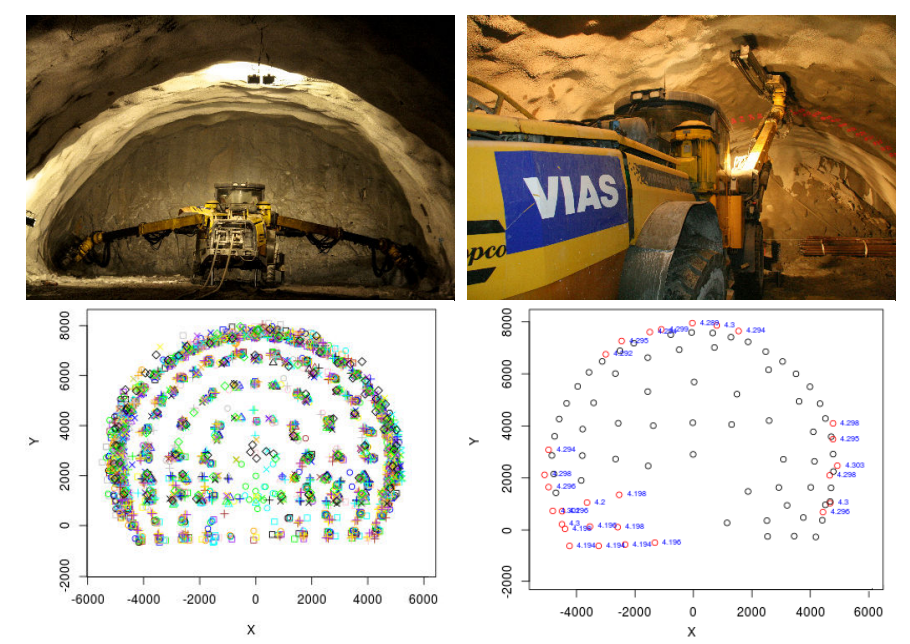

Fig. 1. Drill \& Blast excavation method: excavation front and pattern of drilling holes.

\section{COMPutational InTELligence FOR MWD AND RMR: AN APPROACH}

The current proposal is based on the application of wellknown computational intelligence techniques to an engineering problem concerning how available MWD data can be used during tunnel excavation to give high level support. The issues of this approach are based on ML\&CI techniques, involving from data analysis to prediction and decision making, as well as the extraction of a knowledge base. Clearly and methodologically defining the stages to be dealt with in order to take advantage of the MWD data using ML\&CI for the estimation of operational tunnel parameters, and how every stage can be implemented by these techniques.

In Figure 2, the general scheme of this methodology, as well as its key issues, are described. The major goal of this work is the prediction of tunnel design parameters, the current version concerns the RMR:

1) Stage 1 - Unsupervised Variable Selection: the MWD data available is processed in order to validate data, removing outliers, fixing missing values, etc. Then a 


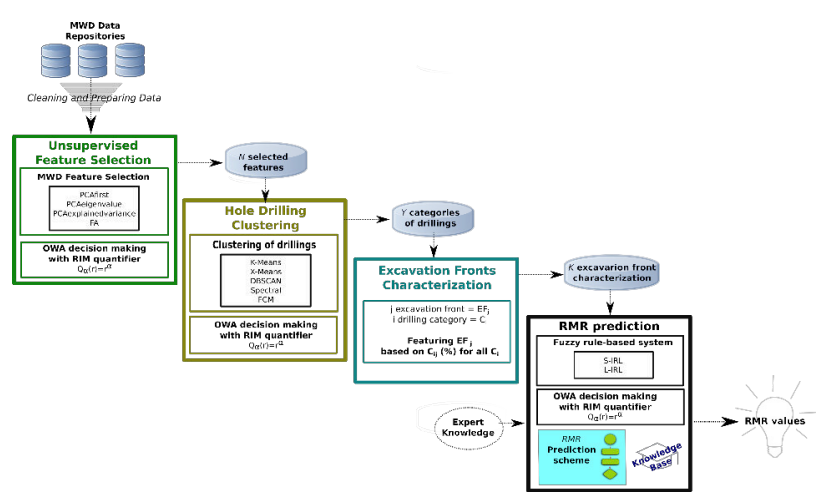

Fig. 2. Main stages of the Proposal

selection of the most relevant variables is carried out based on two well-known unsupervised techniques: PCA and FA. Finally, an OWA based multicriteria decision making is carried out to obtain an unsupervised ranking with the most relevant MWD variables to be used in the following stages.

2) Stage 2 - Hole Drilling Clustering: considering the previous MWD variable selection, different clustering algorithms and indexes are used to validate each alternative clustering or partitioning. Different categories of hole drillings are obtained and described based on the MWD variables. Once again, based on alternative clustering categories and performances, an OWA based multicriteria decision making is carried out to provide a ranking of the parameters for the clustering algorithms involved.

3) Stage 3 - Characterization of Excavation Fronts: based on the hole drilling categories obtained in the previous stage, every excavation front is characterized and summarized through its own distribution of MWD based hole drilling categories.

4) Stage 4 - Prediction of RMR Values and Weightings: based on linguistic and scatter fuzzy systems, as well as the expert knowledge collected, the prediction of the RMR values are set out. The best prediction model is based on an OWA decision making applied over all the alternative fuzzy models and their performances from different points of view, such as error, complexity or number of linguistic variables and terms. Besides, the fuzzy nature of these algorithms permits a knowledge base to be obtained, which is expressed by (linguistic) fuzzy rules, linking the MWD data with the expert knowledge available.

The on-site predictions and in advance, of the RMR values are extremely valuable for the technicians to manage uncertainties and plan the pattern of hole drillings for the new excavation front ahead, as well as the support needed for the tunnel walls. These estimations allow extra support to minimize risks in the advance of the tunnel.

\section{SUMMARY OF RESULTS}

The open approach introduced in this paper is able to manage, on-site, the MWD data generated by the drill rig to
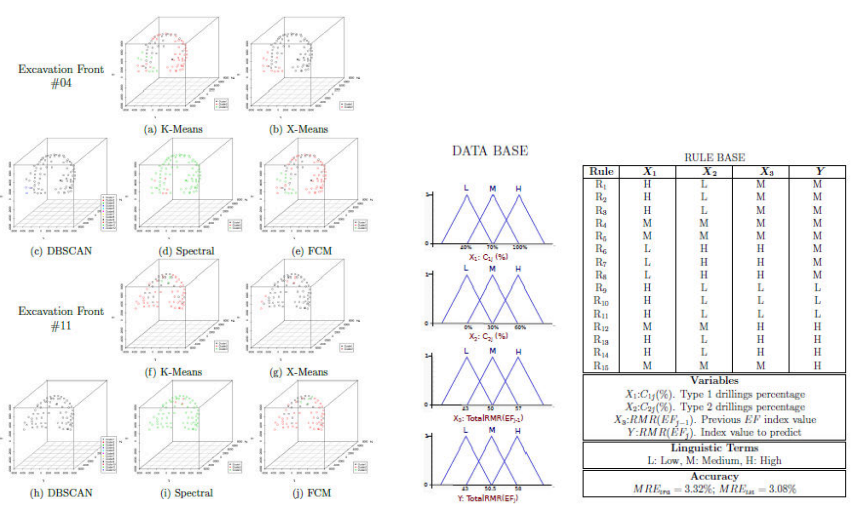

Fig. 3. Cluster based excavation front and Base of Fuzzy Rules

estimate the critical RMR value of every excavation front of the tunnel in progress. The analysis of the MWD data, on the time and frequency domain, has permitted only 3 main MWD features to be used as the basis for the rest of the methodology, meaning a huge reduction in the complexity of the solution.

This reduced number permits the drillings for a clustering procedure to be featured. In this way, every excavation front is summarized by a very few features based on these MWD drilling based rocky categories. This can be seen as a feature extraction that summarizes the characterization of every excavation front to an affordable dimensionality for ML\&CI approaches. These reductions of dimensionality/complexity are critical for addressing this challenge.

Most different cluster policies, or algorithms, have shown that 2 or 3 clusters is a well-balanced number of MWD drilling based rocky categories. This fits with the knowledge and expertise concerning this issue of the technicians in charge of this type of work. This characterization has permitted the RMR value to be estimated using a linguistic and scatter FRBS: permitting the capability of both different approaches to be checked, so as to estimate the RMR while also generating a reasonable base of well-balanced fuzzy rules regarding accuracy-interpretability. This means being able to generate a good estimation and an "interpretable" knowledge base about the drilling features of every excavation front and their RMR values based on linguistic terms. This modelling has been made possible by the expert knowledge provided by geologists concerning the excavation fronts. The linguistic approach (LIRL) has been slightly better than the scatter option (SIRL), providing the best approach for RMR estimation as a reasonable base of knowledge: $M R E_{t s t}=3.01 \%, 17$ fuzzy rules, 3 linguistic variables and 3 linguistic terms (Fig. 3). So the complexity of this knowledge base is affordable. Other more accurate predictions are possible, $M R E_{t s t}=3.01 \%$, but with an increase in the complexity, 83 fuzzy rules.

\section{REFERENCES}

[1] M. Galende, M. M.J. Fuente, G. Sainz-Palmero, M. Menéndez, Monitor-while-drilling - based estimation of rock mass rating with computational intelligence: the case of tunnel excavation front, Automation in Construction (93C) (2018) 325-338. doi:https://doi.org/10.1016/j.autcon.2018.05.019. 